\title{
Structural Equivalence and ET0L Grammars $^{\S}$
}

\author{
Kai Salomaa, Derick Wood’, and Sheng $\mathrm{Yu}^{\ddagger}$ \\ Technical Report HKUST-CS95-44 \\ September 1995 \\ *Department of Mathematics \\ University of Turku \\ Turku \\ Finland \\ †Department of Computer Science \\ Hong Kong University of Science \& Technology \\ Clear Water Bay, Kowloon \\ Hong Kong \\ ‡Department of Computer Science \\ University of Western Ontario \\ London, Ontario \\ Canada
}

\begin{abstract}
For a given context-sensitive grammar $G$ we construct ET0L grammars $G_{1}$ and $G_{2}$ that are structurally equivalent if and only if the language generated by $G$ is empty, which implies that structural equivalence is undecidable for ET0L grammars. In contrast, structural equivalence is decidable for E0L grammars and for extended E0L grammars. In fact, we show that structural equivalence is undecidable for propagating ET0L grammars in which the number of tables is restricted to be at most two. A stronger notion of equivalence that requires the sets of syntax trees to be isomorphic is shown to be decidable for ET0L grammars.
\end{abstract}

\footnotetext{
$\S$ This research was supported by grants from the Natural Sciences and Engineering Research Council of Canada and from the Information Technology Research Centre of Ontario. 


\title{
Structural Equivalence and ET0L Grammars ${ }^{1}$
}

\author{
Kai Salomaa ${ }^{2} \quad$ Derick Wood ${ }^{3} \quad$ Sheng $\mathrm{Yu}^{4}$
}

September 12, 1995

\begin{abstract}
For a given context-sensitive grammar $G$ we construct ET0L grammars $G_{1}$ and $G_{2}$ that are structurally equivalent if and only if the language generated by $G$ is empty, which implies that structural equivalence is undecidable for ET0L grammars. In contrast, structural equivalence is decidable for E0L grammars and for extended E0L grammars. In fact, we show that structural equivalence is undecidable for propagating ET0L grammars in which the number of tables is restricted to be at most two. A stronger notion of equivalence that requires the sets of syntax trees to be isomorphic is shown to be decidable for ET0L grammars.
\end{abstract}

\section{Introduction}

When considering various devices such as grammars and automata for defining languages, a central question is to determine whether two such devices are equivalent; that is, whether they generate (or recognize) the same language. It is well known that language equivalence is undecidable for contextfree and E0L grammars, since two grammars may, in general, be language equivalent although the derivations of a given sentence are completely different. When dealing with sequential or parallel context-free grammars, we may consider the notion of structural equivalence, also known as strong equivalence, instead of language equivalence. Two grammars are structurally equivalent if the structures of the syntax trees that correspond to each sentence are the same. We define the structure of a syntax tree as the tree that is obtained by deleting the nonterminals that label internal nodes. An even stronger notion of equivalence, which we call syntax equivalence, requires that the sets of syntax trees are identical modulo a renaming of the nonterminal symbols.

Paull and Unger [9], and McNaughton [5] showed that structural equivalence of context-free grammars is decidable. Thatcher [15, 16] gave a considerably simpler proof of decidability by reducing it to the emptiness problem of finite-state tree automata. Ginsburg and Harrison [2] established the decidability of a more restricted problem, namely, they encoded the syntax trees of a

\footnotetext{
${ }^{1}$ This research was supported by grants from the Natural Sciences and Engineering Research Council of Canada and by a grant from the Information Technology Research Centre of Ontario.

${ }^{2}$ Department of Mathematics, University of Turku, Turku, Finland.

${ }^{3}$ Contact author: Department of Computer Science, Hong Kong University of Science \& Technology, Clear Water Bay, Kowloon, Hong Kong.

${ }^{4}$ Department of Computer Science, University of Western Ontario, London, Ontario, Canada N6A 5B7.
} 
context-free grammar as a bracketed context-free language and showed that equivalence of bracketed languages is decidable. Thatcher [15] also established decidability of structural equivalence for extended context-free grammars ("context-free grammars" that have productions with regular righthand sides), and Cameron and Wood $[1,18]$ give a grammatical proof of decidability that is similar to McNaughton's proof for context-free grammars as expounded by Salomaa [12].

The question of structural equivalence for E0L grammars was first raised by Ottmann and Wood $[7,8]$, where they also obtained partial decidability results for certain restricted types of grammars. E0L structural equivalence was shown to be decidable by Salomaa and Yu [14] using the automata-theoretic approach of Thatcher $[15,16]$. The same proof can be used to see that syntax equivalence of E0L grammars is also decidable. An alternative grammatical proof for the decidability of E0L structural equivalence was given by Niemi [6] based on the approach of Ottmann and Wood [8]. The grammatical proof is more complicated (as in the case of context-free grammars), but it has the advantage that it produces for a given E0L grammar a structurally equivalent normal form such that two E0L grammars in the normal form are structurally equivalent if and only if they are isomorphic. This decidability result has been extended by Cameron and Wood [1, 18] to extended E0L grammars ("E0L grammars" that have productions with regular right-hand sides). The complexity of the E0L structural equivalence problem has been studied by Salomaa et al. [13]. Recently, Istrate [3] has shown that structural equivalence of ET0L grammars is decidable when we require that corresponding syntax trees use isomorphic sequences of tables. The decidability of structural equivalence for T0L and EDT0L grammars remains open. We conjecture that T0L structural equivalence is decidable since every level in two structurally equivalent syntax trees must give identical terminal strings. We cannot relabel the internal nodes of a syntax tree as we do for ET0L syntax trees. It was noted by Salomaa and Yu [14] that structural equivalence is undecidable for indexed grammars.

Here we show that structural equivalence is undecidable for ET0L grammars. More specifically, structural equivalence is already undecidable for a propagating E0L grammar and a propagating ET0L grammar. Furthermore, the number of tables in the ET0L grammar can be restricted to two. In contrast we show that syntax equivalence is decidable for ET0L grammars. These results demonstrate that the notions of structural and syntax equivalence are essentially different.

The proof of undecidability uses a reduction from the emptiness problem for context-sensitive languages, which is a well known undecidable problem $[12,17]$. For a given context-sensitive grammar $G$, we construct ET0L grammars $G_{1}$ and $G_{2}$ that are structurally equivalent if and only if $L(G)=\emptyset$. The construction is considerably simplified by the use of a normal form for contextsensitive grammars in which the productions have only one-sided context that was established by Penttonen [10].

Intuitively, the grammar $G_{1}$ simulates the context-sensitive grammar $G$ by ignoring the context conditions. For technical reasons we add new branches to the derivations in $G_{1}$ that we use to guarantee that the syntax trees are in one-to-one correspondence with their structures. The grammar $G_{2}$ simulates $G$ in a similar way but, in addition, it nondeterministically verifies that the context conditions of $G$ are violated at least once. Intuitively, $G_{2}$ uses a context-sensitive production once, which can be accomplished by sending, nondeterministically, messages down the 
syntax tree. The choice of table that is used to delete the messages gives the necessary context information for the context-sensitive derivation step.

\section{Preliminaries}

We assume that the readers are familiar with the basics of formal language theory and with ET0L grammars in particular $[11,12,17]$. In the following, we review the definitions of the syntax trees of ET0L grammars.

Let $A$ be a finite set. The cardinality of $A$ is denoted $\# A$ and the power set of $A$ is $\mathcal{P}(A)$. The family of finite multisets over $A$ is $\mathcal{M}(A)$. A multiset is denoted by listing its elements in double braces. Let $a \in A$ and $B=\left\{\left\{b_{1}, \ldots, b_{m}\right\}\right\} \in \mathcal{M}(A)$; then, $\#_{a}[B]$ denotes the number of occurrences of $a$ in the sequence $b_{1}, \ldots, b_{m}$. Also,

$$
\operatorname{base}(B)=\left\{a \in A: \#_{a}[B] \geq 1\right\} .
$$

The set of finite strings (resp. nonempty finite strings) over $A$ is $A^{*}$ (resp. $A^{+}$). The empty string is denoted by $\lambda$. For $a \in A$ and $w \in A^{*}$, $\#_{a}(w)$ denotes the number of occurrences of $a$ in the string $w$. Also we define

$$
\operatorname{alph}(w)=\left\{a \in A: \#_{a}(w) \geq 1\right\} .
$$

An ETOL grammar $G$ is specified by a tuple

$$
G=(V, \Sigma, S, H),
$$

where $V$ is a finite alphabet of nonterminals, $\Sigma$ is a finite alphabet of terminals, $S \in V$ is the initial nonterminal, and $H$ is a finite set of tables of productions from $V$ to finite subsets of $(V \cup \Sigma)^{*}$. We define a table $h \in H$ as a finite set of productions $a \rightarrow w$, where $a \in V$ and $w \in(V \cup \Sigma)^{*}$. A grammar $G$ is an E0L grammar if it has only one table, that is, $\# H=1$. We say that a grammar is propagating if the right side of every production is nonempty, that is, for all $h \in H, a \in V$ : $(a \rightarrow \lambda) \notin h$. Propagating ET0L and E0L grammars are called EPT0L and EP0L grammars, respectively. Although we can restrict our attention to EPT0L grammars for the undecidability of ET0L structural equivalence, for the decidability of syntax equivalence we need to deal with ET0L grammars to obtain the strongest result.

In the following, $G$ denotes an ET0L grammar as in (1). Let $F_{G}$ be the set of all rooted ordered trees where the nodes are labeled by elements of $V \cup \Sigma \cup\{\hat{\lambda}\}$. Here $\hat{\lambda}$ is a new symbol corresponding to the empty string $\lambda$. The set of nodes of $T \in F_{G}$ is denoted as $\operatorname{dom}(T)$, the domain of $T$. The label function associating an element of $V \cup \Sigma \cup\{\hat{\lambda}\}$ to each node of $T$ is denoted by $\operatorname{lab}_{T}: \operatorname{dom}(T) \rightarrow V \cup \Sigma \cup\{\hat{\lambda}\}$.

We define the parallel derivation relation $\rightarrow_{G}^{\mathrm{par}} \subseteq F_{G} \times F_{G}$ as follows. Let $T, T^{\prime} \in F_{G}$. Then $T \rightarrow{ }_{G}^{\text {par }} T^{\prime}$ if and only if $T^{\prime}$ is obtained from $T$ as follows. Assume that $T$ has $n$ external nodes $u_{1}, \ldots, u_{n}$ where $\operatorname{lab}_{T}\left(u_{i}\right)=a_{i} \in V \cup\{\hat{\lambda}\}, i=1, \ldots, n$. Consider a table $h \in H$ and for every $i \in\{1, \ldots, n\}$ such that $a_{i} \neq \hat{\lambda}$ choose a production

$$
a_{i} \rightarrow b_{1}^{i} \cdots b_{k_{i}}^{i} \in h,
$$


$b_{j}^{i} \in V \cup \Sigma, j=1, \ldots k_{i}, k_{i} \geq 0$. If $a_{i} \neq \hat{\lambda}$ and $k_{i} \geq 1$, then in $T^{\prime}$ the node $u_{i}$ has $k_{i}$ successors labeled respectively by the symbols $b_{1}^{i}, \ldots, b_{k_{i}}^{i}$. If $a_{i} \neq \hat{\lambda}$ and $k_{i}=0$, then the node $u_{i}$ has exactly one successor labeled by the symbol $\hat{\lambda}$. If $a_{i}=\hat{\lambda}$, then $u_{i}$ has no successors in $T^{\prime}$.

The set of syntax trees $S(G)$ of an ET0L grammar $G$ is defined by

$$
S(G)=\left\{T \in F_{G}: S^{\prime}\left(\rightarrow_{G}^{\mathrm{par}}\right)^{*} T\right\},
$$

where $S^{\prime}$ is the tree with a single node labeled by $S$.

In a syntax tree $T$ all paths from the root to an external node labeled by an element of $V U$ $\Sigma$ have the same length. Note that a path from the root to a node labeled with $\hat{\lambda}$ need not be the same length as the paths from the root to nodes labeled with elements of $V \cup \Sigma$. In an EPT0L grammar, however, all root-to-external-node paths as the same length. According to our definition, if a tree $T \in S(G)$ has an external node labeled by a terminal symbol, then the derivation cannot be continued from $T$, that is, $G$ is synchronized, see the text of Rozenberg and Salomaa [11]. We observe that the assumption of syncronization does not affect our results. Given a nonsynchronized ET0L grammar, we can convert it into an equivalent synchronized ET0L grammar by introducing a new nonterminal symbol, a nonterminal partner, for each terminal symbol in the grammar. Next, we replace every appearance of a terminal symbol in the productions of the grammar with its partner and, finally, add to each table a production that rewrites each nonterminal partner as its corresponding terminal symbol. Clearly, every terminal syntax tree in the nonsynchronized grammar corresponds to a terminal syntax tree in the synchronized grammar that has an extra last level that uses the added productions and conversely. Thus, based on this construction, two nonsynchronized ET0L grammars are structurally equivalent if and only if their synchronized versions are structurally equivalent. Alternatively, since nonsynchronized ET0L grammars are a wider class than synchronized grammars, the undecidablity carries over directly.

A syntax tree $T \in S(G)$ is terminal if all external nodes of $T$ are labeled by elements of $\Sigma \cup\{\hat{\lambda}\}$. The set of terminal syntax trees of $G$ is denoted by $T S(G)$.

Let $e_{\lambda}:(V \cup \Sigma \cup\{\hat{\lambda}\})^{*} \rightarrow(V \cup \Sigma)^{*}$ be the morphism defined by the conditions $e_{\lambda}(a)=a$ if $a \in V \cup \Sigma$ and $e_{\lambda}(\hat{\lambda})=\lambda$. For $T \in F_{G}$ denote by $w_{T}\left(\in(V \cup \Sigma \cup\{\hat{\lambda}\})^{+}\right)$the string obtained by catenating from left to right the symbols labeling the external nodes of $T$. The yield of $T$ is defined as

$$
\operatorname{yield}(T)=e_{\lambda}\left(w_{T}\right)
$$

The set of sentential forms of an ET0L grammar $G$ is

$$
\operatorname{sf}(G)=\{\operatorname{yield}(T): T \in S(G)\} .
$$

The relation $\rightarrow \rightarrow_{G}^{\mathrm{par}}$ determines a parallel derivation relation $\Rightarrow_{G}^{\mathrm{par}}$ on $(V \cup \Sigma)^{*}$ as follows. Let $w_{1}, w_{2} \in(V \cup \Sigma)^{*}$. Then $w_{1} \Rightarrow_{G}^{\mathrm{par}} w_{2}$ if and only if there exist $T_{i} \in F_{G}, i=1,2$, with yield $\left(T_{i}\right)=w_{i}$ such that $T_{1} \rightarrow{ }_{G}^{\text {par }} T_{2}$. The language generated by $G$ is

$$
L(G)=\operatorname{sf}(G) \cap \Sigma^{*}=\left\{w \in \Sigma^{*}: S\left(\Rightarrow_{G}^{\mathrm{par}}\right)^{*} w\right\} .
$$

Clearly the preceding definition of $L(G)$ is equivalent to the standard definition of the language generated by an ET0L grammar given by Rozenberg and Salomaa [11]. 
Let $T \in T S(G)$. The structure of the terminal syntax tree $T, \operatorname{str}_{G}(T)$, is the external-nodelabeled tree obtained from $T$ by removing the labels of all internal nodes of $T$ (that is, nodes that are not external). Formally, $\operatorname{str}_{G}(T)=T^{\prime}$ can be defined as follows. Let $c$ be a new symbol not in $V \cup \Sigma$. Then $\operatorname{dom}\left(T^{\prime}\right)=\operatorname{dom}(T), \operatorname{lab}_{T^{\prime}}(u)=\operatorname{lab}_{T}(u)$ if $u$ is an external node of $T$ and $\operatorname{lab}_{T^{\prime}}(u)=c$ if $u$ is an internal node of $T$. We denote

$$
S T S(G)=\left\{\operatorname{str}_{G}(T): T \in T S(G)\right\} .
$$

Now we can define the various notions of equivalence of grammars considered here. Let $G_{1}$ and $G_{2}$ be ET0L grammars. The grammars $G_{1}$ and $G_{2}$ are said to be

- language equivalent if $L\left(G_{1}\right)=L\left(G_{2}\right)$,

- structurally equivalent if $S T S\left(G_{1}\right)=S T S\left(G_{2}\right)$, and

- syntax equivalent if $T S\left(G_{1}\right)$ and $T S\left(G_{2}\right)$ are equal modulo a renaming of the nonterminals.

Note that syntax equivalent grammars are always structurally equivalent and structural equivalence in turn implies language equivalence. It is well known that language equivalence is undecidable already for context-free grammars. Structural equivalence of context-free and E0L grammars is decidable $[5,6,8,9,14,15,16]$. Syntax equivalence of context-free grammars is considered in Ginsburg and Harrison [2].

To conclude this section we recall the definition of a normal form for context-sensitive grammars established by Penttonen [10]. A PNF (Penttonen-normal-form) context-sensitive grammar is specified by a tuple $G_{P N F}=\left(U_{N}, U_{T}, I, P\right)$, where $U_{N}$ is a finite set of nonterminals, $U_{T}$ is a finite set of terminals, $I \in U_{N}$ is the initial nonterminal, and $P$ is a set of productions of the following three types:

- Right-context productions: $B D \rightarrow C D$, where $B, C, D \in U_{N}$

- Context-free productions: $B \rightarrow C D$, where $B, C, D \in U_{N}$

- Terminating productions: $B \rightarrow b$, where $B \in U_{N}, b \in U_{T}$

Thus, we allow only one-sided context in the productions. The productions of $P$ define, in a natural way, the (sequential) rewrite-relation $\Rightarrow_{G_{P N F}} \subseteq\left(U_{N} \cup U_{T}\right)^{+} \times\left(U_{N} \cup U_{T}\right)^{+}$and the language generated by $G_{P N F}$ is

$$
L\left(G_{P N F}\right)=\left\{w \in U_{T}^{+}: I \Rightarrow_{G_{P N F}}^{+} w\right\} .
$$

Strictly speaking, instead of the preceding productions with a right context condition Penttonen normal form [10] allows only left context in the productions of the grammar, (that is, productions of the form $D B \rightarrow D C$.) The definitions are, however, completely symmetric. Penttonen [10] proved the following result.

Theorem 2.1 (Penttonen [10]) For an arbitrary context-sensitive grammar $G_{C S}$ (with no length reducing productions) we can effectively construct a $P N F$ grammar $G_{P N F}$ such that $L\left(G_{P N F}\right)=$ $L\left(G_{C S}\right)$. 


\section{Syntax equivalence}

For context-free and E0L grammars both syntax equivalence and structural equivalence are decidable $[2,5,6,14]$. Before proving our main undecidability result, we show that syntax equivalence is decidable for ET0L grammars.

Lemma 3.1 Given ETOL grammars $G_{i}=\left(V_{i}, \Sigma_{i}, S_{i}, H_{i}\right), i=1,2$, we can effectively decide whether

$$
T S\left(G_{1}\right)=T S\left(G_{2}\right)
$$

Proof. We say that an ET0L grammar $G$ is reduced if all nonterminal and terminal symbols of $G$ appear in some terminal syntax tree of $G$. Using standard methods we can effectively find the subsets $V_{i}^{\prime} \subseteq V_{i}, \Sigma_{i}^{\prime} \subseteq \Sigma_{i}, 1 \leq i \leq 2$, that consist of all symbols appearing in some tree $T \in T S\left(G_{i}\right)$. Thus, we can also effectively construct a reduced grammar $G_{i}^{\prime}$ that is syntax equivalent to $G_{i}$, for $i=1,2$, simply by removing the unnecessary symbols of $V_{i} \cup \Sigma_{i}$ and the productions that contain some of these symbols. Hence, without loss of generality, we can assume that the grammars $G_{1}$ and $G_{2}$ are reduced and that $V_{1}=V_{2}=V, \Sigma_{1}=\Sigma_{2}=\Sigma, S_{1}=S_{2}=S$, because if, say, $V_{1} \neq V_{2}$ and $G_{1}$ and $G_{2}$ are reduced, then $\operatorname{TS}\left(G_{1}\right) \neq T S\left(G_{2}\right)$.

The proof is based on the straightforward observation that $T S\left(G_{1}\right) \subseteq T S\left(G_{2}\right)$ if and only if, for every set of productions $p_{1}, \ldots, p_{m} \in h, h \in H_{1}$, that can be used in one parallel step of a successful derivation of $G_{1}$, there exists a table $h^{\prime} \in H_{2}$ such that $p_{1}, \ldots, p_{m} \in h^{\prime}$. We define a family $\gamma_{1}$ of multisets over $V \cup \Sigma$ that determines which sets of productions of $H_{1}$ are simultaneously applicable in a derivation starting from the initial nonterminal. Also, we define a collection $\eta_{1}$ of sets over $V \cup \Sigma$ that determines which sets of productions of $H_{1}$ yield a sentential form that can eventually be rewritten to a terminal string or sentence. (Note that, although $G_{1}$ is reduced, it is still possible that, for productions $a_{i} \rightarrow w_{i}, i=1,2$, belonging to a table of $H_{1}$, the string $w_{1} w_{2}$ cannot yield a sentence.) Then, to complete the proof it is sufficient to show that the sets $\gamma_{1}, \eta_{1}$ (and the corresponding sets $\gamma_{2}, \eta_{2}$ constructed for the grammar $G_{2}$ ) are recursive. We now give the details of the proof.

For $h \in H_{i}, 1 \leq i \leq 2$, we denote by $M_{h}$, the maximal number of productions of $h$ that have the same left-hand side $a \in V$. Then, we define

$$
M=\max \left\{M_{h}: h \in H_{i}, 1 \leq i \leq 2\right\} .
$$

We say that $w \in(V \cup \Sigma)^{*}$ covers a multiset $B \in \mathcal{M}(V \cup \Sigma)$ if

- $\operatorname{alph}(w)=\operatorname{base}(B)$, and

- $(\forall a \in V \cup \Sigma) \#_{a}(w) \geq \#_{a}[B]$.

Intuitively, if $w$ covers $B$, then $w$ consists of exactly those symbols that belong to $B$ and the multiplicity of each symbol $a$ in $B$ is at most the number of occurrences of $a$ in the string $w$.

Let $\Omega_{M}$ consist of all multisets $B \in \mathcal{M}(V \cup \Sigma)$ such that

$$
(\forall a \in V \cup \Sigma) \#_{a}[B] \leq M .
$$


For $i=1,2$, we define a family $\gamma_{i} \subseteq \mathcal{M}(V \cup \Sigma)$ of multisets as:

$$
\gamma_{i}=\left\{B: B \in \Omega_{M} \text { and }\left(\exists w \in \operatorname{sf}\left(G_{i}\right)\right) \text { such that } w \text { covers } B\right\}
$$

The collection $\gamma_{i}$ of multisets can be effectively constructed. The family $\Omega_{M}$ is finite and, for a given multiset $B \in \Omega_{M}$, we can determine whether $B \in \gamma_{i}$ as follows. Denote by $L_{B}$ the set $\left\{w \in(V \cup \Sigma)^{*}: w\right.$ covers $\left.B\right\}$. Clearly, $L_{B}$ is a regular language; thus, we can construct an ET0L grammar $G_{i}^{B}$ such that

$$
L\left(G_{i}^{B}\right)=\operatorname{sf}\left(G_{i}\right) \cap L_{B} .
$$

To decide whether $B \in \gamma_{i}$, we merely check whether $L\left(G_{i}^{B}\right)$ is nonempty. Recall that the emptiness problem for ET0L grammars is decidable [4].

Next, for $i=1,2$, we define $\eta_{i} \subseteq \mathcal{P}(V \cup \Sigma)$ by

$$
\eta_{i}=\left\{\left\{a_{1}, \ldots, a_{m}\right\}:\left(\exists w \in \Sigma^{*}\right) a_{1} \cdots a_{m}\left(\Rightarrow_{G_{i}}^{\mathrm{par}}\right)^{*} w \text { and } a_{1}, \ldots, a_{m} \in V \cup \Sigma\right\} .
$$

Note that, for $A \in \mathcal{P}(V \cup \Sigma)$, the relation $A \in \eta_{i}$ depends only on the set $A$ although condition (2) contains a sequence of elements of $A$. Similar to the preceding argument, using the decidability of emptiness for ET0L grammars, we verify that $\eta_{i}$ can be effectively constructed.

Now, for $i=1,2$, define the set $\gamma_{i} \diamond \eta_{i} \subseteq \Omega_{M}$ as:

$$
\gamma_{i} \diamond \eta_{i}=\left\{B: B \in \gamma_{i} \text { and } \operatorname{base}(B) \in \eta_{i}\right\}
$$

Thus, $B \in \gamma_{i} \diamond \eta_{i}$ if and only if there exist $w_{1} \in(V \cup \Sigma)^{*}$ and $w_{2} \in \Sigma^{*}$ such that $w_{1}$ covers $B$ and

$$
S\left(\Rightarrow_{G_{i}}^{\mathrm{par}}\right)^{*} w_{1}\left(\Rightarrow_{G_{i}}^{\mathrm{par}}\right)^{*} w_{2}
$$

It should be clear that, if $\gamma_{1} \diamond \eta_{1} \neq \gamma_{2} \diamond \eta_{2}$, then $T S\left(G_{1}\right) \neq T S\left(G_{2}\right)$. Therefore, we need consider only the case

$$
\gamma_{1} \diamond \eta_{1}=\gamma_{2} \diamond \eta_{2}=\omega
$$

Let $B=\left\{\left\{b_{1}, \ldots, b_{m}\right\}\right\} \in \Omega_{M}$ be such that base $\left.B\right) \subseteq V$. We say that $B$ is (1,2)-consistent if the following condition holds:

For every $h \in H_{1}$ and sequence

$$
p_{1}: b_{1} \rightarrow w_{1}, \ldots, p_{m}: b_{m} \rightarrow w_{m},
$$

of productions of $h$, where $w_{1}, \ldots, w_{m} \in(V \cup \Sigma)^{*}$, such that

$$
\bigcup_{i=1}^{m} \operatorname{alph}\left(w_{i}\right) \in \eta_{1}
$$

there exists $h^{\prime} \in H_{2}$ such that $p_{1}, \ldots, p_{m} \in h^{\prime}$. Note that $B$ is a multiset and the elements $b_{1}, \ldots, b_{m}$ are not necessarily distinct.

We claim that

$$
T S\left(G_{1}\right) \subseteq T S\left(G_{2}\right)
$$


if and only if

$$
(\forall B \in \omega) \quad B \text { is }(1,2) \text {-consistent. }
$$

First assume that (6) holds and let

$$
S=T_{0} \rightarrow \underset{G_{1}}{\mathrm{par}} T_{1} \rightarrow \underset{G_{1}}{\mathrm{par}} \cdots \rightarrow \rightarrow_{G_{1}}^{\mathrm{par}} T_{n}
$$

be the derivation of an arbitrary syntax tree $T_{n} \in T S\left(G_{1}\right)$. Let $j \in\{0, \ldots, n-1\}$ and assume that the derivation step

$$
D_{j}: T_{j} \rightarrow_{G_{1}}^{\mathrm{par}} T_{j+1}
$$

uses a table $h \in H_{1}$. For $a \in V$ denote by $D_{j}(a)$ the number of different productions of $h$ with left side $a$ used in $D_{j}$. Let $B_{j}$ be the multiset containing $D_{j}(a)$ copies of the element $a \in V$. Then yield $\left(T_{j}\right)$ covers $B_{j}$ and it follows that $B_{j} \in \gamma_{1} \diamond \eta_{1}=\omega$. (Note that $B_{j} \in \Omega_{M}$ since $D_{j}(a) \leq M$ for every $a \in V$.) Hence $B_{j}$ is (1,2)-consistent by the assumption (6). Since $\operatorname{yield}\left(T_{j+1}\right)\left(\Rightarrow_{G_{1}}^{\text {par }}\right)^{*}$ yield $\left(T_{n}\right) \in \Sigma^{*}$ it follows that the set of productions of $h$ used in the derivation step $D_{j}$ satisfies the condition (4). Now by the (1,2)-consistency condition there exists $h^{\prime} \in H_{2}$ that can be used to exactly simulate the derivation step $D_{j}, 0 \leq j \leq n-1$. Thus $T_{n} \in T S\left(G_{2}\right)$.

Conversely, assume that $B=\left\{\left\{b_{1}, \ldots, b_{m}\right\}\right\} \in \omega$ is not $(1,2)$-consistent. Then there exists $h \in H_{1}$ and $p_{1}, \ldots, p_{m} \in h$ as in (3) and (4) such that

$$
p_{1}, \ldots, p_{m} \text { are not contained in any table of } H_{2} \text {. }
$$

Since $B \in \omega$, there exists $T \in S\left(G_{1}\right)$ such that yield $(T)$ covers $B$. Let $\left\{u_{1}, \ldots, u_{n}\right\}$ be the set of external nodes of $T$ that are labeled with elements of $V$. Since yield $(T)$ covers $B$, there exists a surjective mapping $f$ from $\left\{u_{1}, \ldots, u_{n}\right\}$ to the multiset $B$ such that $f\left(u_{i}\right)=\operatorname{lab}_{T}\left(u_{i}\right), 1 \leq i \leq n$. Consider the derivation step

$$
D: T \rightarrow_{G_{1}}^{\mathrm{par}} T_{1},
$$

where in an external node $u_{i}, 1 \leq i \leq n$, one applies the production $p_{j}: b_{j} \rightarrow w_{j}$ where $f\left(u_{i}\right)=b_{j}$, $j \in\{1, \ldots, m\}$. Since $f$ is surjective, the derivation step $D$ uses all productions $p_{1}, \ldots, p_{m}$. Also, since $\bigcup_{i=1}^{m}$ alph $\left(w_{i}\right) \in \eta_{1}$, there exists $T_{2} \in T S\left(G_{1}\right)$ such that $T_{1}\left(\rightarrow_{G_{1}}^{\mathrm{par}}\right)^{*} T_{2}$. On the other hand, it is clear that $T_{2} \notin T S\left(G_{2}\right)$. Note that by $(7), T \nrightarrow_{G_{2}}^{\mathrm{par}} T_{1}$.

For a given multiset $B \in \omega$, the $(1,2)$-consistency condition is decidable, since $\eta_{1}$ can be computed. Since $\omega$ is finite and recursive, we can decide whether (5) holds. Finally, by symmetry, we can determine whether $T S\left(G_{2}\right) \subseteq T S\left(G_{1}\right)$.

Since the number of nonterminals is finite the following result follows immediately from Lemma 3.1.

Theorem 3.1 Syntax equivalence is decidable for ETOL grammars.

\section{Structural equivalence}

Here we prove our main result: structural equivalence of ET0L grammars is undecidable. In the proof we need to consider only propagating grammars and, furthermore, one of the grammars can 
be restricted to have only one table. The proof uses a reduction from the emptiness problem for context-sensitive languages, which is well known to be undecidable [12, 17]. For a given PNF context-sensitive grammar $G_{P N F}$ we construct ET0L grammars $G_{1}$ and $G_{2}$ that are structurally equivalent if and only if the language generated by $G_{P N F}$ is empty. The grammar $G_{1}$ essentially simulates the derivation of $G_{P N F}$ but ignores the context conditions. The grammar $G_{2}$ is as $G_{1}$ but, in addition, it sends messengers down the syntax tree that nondeterministically verify that the context condition of $G_{P N F}$ is violated somewhere in the syntax tree.

The construction that we use requires that the productions of the given context-sensitive grammar have only one-sided context, more specifically, right-sided context. (The proof could easily be modified to use left-sided context which is the original normal form of Penttonen.) Because of this restriction, we need only two messengers in the syntax tree. More important, the right-sided context allows us to interpret an arbitrary parallel derivation step of the corresponding ET0L grammar $G_{1}$, $T_{1} \rightarrow{ }_{G_{1}}^{\mathrm{par}} T_{2}$, as a sequence of rewrite steps of $G_{P N F}$, performed from left to right by checking the context conditions only with respect to the initial sentential form, yield $\left(T_{1}\right)$. If we had productions with two-sided context, the construction would be considerably more involved.

Theorem 4.1 Given an EPOL grammar $G_{1}$ and an EPTOL grammar $G_{2}$, it is undecidable whether

$$
\operatorname{STS}\left(G_{1}\right)=\operatorname{STS}\left(G_{2}\right)
$$

Proof. Let $G_{P N F}=\left(U_{N}, U_{T}, I, P\right)$ be an arbitrary PNF context-sensitive grammar. Let

$$
p_{1}, \ldots, p_{k_{1}}, p_{k_{1}+1} \ldots, p_{k}, \quad 0 \leq k_{1} \leq k
$$

be an enumeration of the nonterminating productions of $P$, where $p_{1}, \ldots, p_{k_{1}}$ are the right-context productions and $p_{k_{1}+1}, \ldots, p_{k}$ are the context-free productions of $P$. We construct an EP0L grammar $G_{1}$ and EPT0L grammar $G_{2}$ such that $(8)$ holds if and only if $L\left(G_{P N F}\right)=\emptyset$.

Choose

$$
G_{1}=\left(U_{N} \cup\left\{X, Y, S_{1}\right\}, U_{T} \cup\{\$, @\}, S_{1},\{h\}\right),
$$

where $X, Y, S_{1}$ are new nonterminal symbols, $\$$, @ are new terminal symbols $\left(X, Y, S_{1}, \$, @ \notin U_{N} \cup\right.$ $\left.U_{T}\right)$, and the table $h$ contains exactly the following productions:

$\left(G_{1} \cdot \mathbf{0}\right) S_{1} \rightarrow I Y$.

$\left(G_{1} .1\right) B \rightarrow C X^{i}$ if $p_{i}: B D \rightarrow C D, 1 \leq i \leq k_{1}$, is a right-context production of $P$.

$\left(G_{1} .2\right) B \rightarrow C D X^{i}$ if $p_{i}: B \rightarrow C D, k_{1}<i \leq k$, is a context-free production of $P$.

$\left(G_{1} .3\right) B \rightarrow B$ for every $B \in U_{N}$.

$\left(G_{1} .4\right) B \rightarrow b$ if $B \rightarrow b$ is a terminating production of $P$.

$\left(G_{1} .5\right) X \rightarrow X, X \rightarrow \$, Y \rightarrow Y, Y \rightarrow @$. 
Intuitively, the grammar $G_{1}$ simulates the derivations of $G_{P N F}$ by ignoring the context conditions: the productions defined in $\left(G_{1} .1\right)$ can be applied independently of the right context. In addition, the grammar $G_{1}$ adds, for each nonterminating production $p_{i}$, $i$ copies of the nonterminal $X$ to the derivation tree. This technical modification ensures that a terminal syntax tree $T \in T S\left(G_{1}\right)$ is always uniquely determined by $\operatorname{str}_{G_{1}}(T)$. Also, for technical reasons, the production $\left(G_{1} .0\right)$ introduces a "right endmarker" $Y$ in the derivation. Note that the initial nonterminal $S_{1}$ appears only at the root of each syntax tree of $G_{1}$.

Claim 1. The function $\operatorname{str}_{G_{1}}: T S\left(G_{1}\right) \rightarrow S T S\left(G_{1}\right)$ is injective.

Proof of Claim 1. Let $T_{1}, T_{2} \in T S\left(G_{1}\right)$ be such that $\operatorname{str}_{G_{1}}\left(T_{1}\right)=\operatorname{str}_{G_{1}}\left(T_{2}\right)=t$. The tree $T_{i}$, $i=1,2$, is determined completely by the domain $\operatorname{dom}\left(T_{i}\right)$ and the label function $\operatorname{lab}_{T_{2}}$. Since $\operatorname{str}_{G_{1}}\left(T_{1}\right)=\operatorname{str}_{G_{1}}\left(T_{2}\right)$, it follows that $\operatorname{dom}\left(T_{1}\right)=\operatorname{dom}\left(T_{2}\right)=\operatorname{dom}(t)$ and $\operatorname{lab}_{T_{1}}(u)=\operatorname{lab}_{T_{2}}(u)$, for every external node $u$. We show that, for every internal node $u \in \operatorname{dom}(t), \operatorname{lab}_{T_{1}}(u)=\operatorname{lab}_{T_{2}}(u)$ by induction on the distance $d(u)$ of $u$ from the root of $t$.

(i) If $d(u)=0$ or $d(u)=1$, then $\operatorname{lab}_{T_{i}}(u)$ is uniquely determined by the production $\left(G_{1} .0\right)$, $1 \leq i \leq 2$.

(ii) Let $u$ be an internal node of $t$ that is not the root and let $d(u)=e \geq 2$. Let $v$ be the parent of $u$ and let $\tilde{y}=\left(u_{1}, \ldots, u_{m}\right), m \geq 1$, be the sequence of children of $v$, where $u_{r}=u$, for some $r \in\{1, \ldots, m\}$. Let $\tilde{z}=\left(u_{j+1}, \ldots, u_{m}\right), 0 \leq j \leq m$, be the subsequence of $\tilde{y}$ that consists of all nodes $u_{i}$ that are the roots of unary trees that have external nodes labeled with $\$$. (From the definition of the productions of $G_{1}$ it follows that $\tilde{z}$ is necessarily a suffix of $\tilde{y}$.)

(a) If $\tilde{z}$ is the empty sequence, then necessarily $m=1$ and the production that is applied at the node $v$ in the syntax tree $T_{i}, 1 \leq i \leq 2$, has to be of the forms $B \rightarrow B$, $B \in U_{N}$, or $Y \rightarrow Y$. By the inductive assumption $\operatorname{lab}_{T_{1}}(v)=\operatorname{lab}_{T_{2}}(v)$ and it follows that $\operatorname{lab}_{T_{1}}(u)=\operatorname{lab}_{T_{2}}(u)$.

(b) If $\tilde{y}=\tilde{z}$, then necessarily $m=1$ and the production that is applied at $v$ in $T_{i}, 1 \leq i \leq 2$, has to be $X \rightarrow X$.

(c) If $\tilde{y} \neq \tilde{z}$ and $\tilde{z}$ is nonempty, then $1 \leq j<m$. Clearly, for $i \in\{1,2\}$ and $n \in\{1, \ldots, m\}$, $\operatorname{lab}_{T_{i}}\left(u_{n}\right)=X$ if and only if $n>j$. (The external nodes of the subtrees that correspond to the nodes $u_{j+1}, \ldots, u_{m}$ are labeled by the terminal symbol $\$$.) Thus, the production applied at the node $v$ in $T_{i}, 1 \leq i \leq 2$, has to be the production that corresponds (by $\left(G_{1} .1\right)$ or $\left.\left(G_{1} .2\right)\right)$ to the production $p_{m-j}$ of the grammar $G_{P N F}$. It follows that $\operatorname{lab}_{T_{1}}\left(u_{n}\right)=\operatorname{lab}_{T_{2}}\left(u_{n}\right)$, for all $n \in\{1, \ldots, m\}$.

We have completed the proof of the claim.

We say that a terminal syntax tree $T \in T S\left(G_{1}\right)$ is context-sensitive if the rewrite steps that are indicated in $T$ do not violate the context conditions of the grammar $G_{P N F}$.

More formally, we define the context-sensitive derivation relation (of $G_{1}$ with respect to $G_{P N F}$ ) on the set of syntax trees, $\rightarrow{ }_{G_{1}[C S]}^{\mathrm{par}} \subseteq \rightarrow \underset{G_{1}}{\mathrm{par}}$, as follows. Let $T_{1}, T_{2} \in F_{G_{1}}$ and $T_{1} \rightarrow{ }_{G_{1}}^{\mathrm{par}} T_{2}$. Assume 
that the sequence of external nodes of $T_{1}$ from left to right is $\left(u_{1}, \ldots, u_{m}\right)$ where $u_{i}$ is labeled by $A_{i} \in U_{N} \cup\left\{X, Y, S_{1}\right\}, i=1, \ldots, m$. (Note that $G_{1}$ is propagating.) Furthermore, assume that $T_{2}$ is obtained from $T_{1}$ by attaching $r_{i} \geq 1$ successors labeled by the symbols $B_{1}^{i}, \ldots, B_{r_{i}}^{i}$ to the node $u_{i}$. Then,

$$
T_{1} \rightarrow \underset{G_{1}[C S]}{\mathrm{par}} T_{2}
$$

if and only if the following condition holds.

(CS) Let $i \in\{1, \ldots, m\}$ be such that

$$
B_{1}^{i} \cdots B_{r_{i}}^{i}=C X^{r_{i}-1}, C \in U_{N}, 2 \leq r_{i} \leq k_{1}+1 .
$$

This means that the production $A_{i} \rightarrow B_{1}^{i} \cdots B_{r_{i}}^{i}$ of $h$ corresponds to the right-context production $p_{r_{i}-1}: A_{i} D \rightarrow C D$ of $P$ for some $D \in U_{N}$. Then, there exists $j \in\{i+1, \ldots, m\}$ such that $A_{i+1}=A_{i+2}=\cdots=A_{j-1}=X$ and $A_{j}=D$; that is, the next nonterminal symbol different from $X$ in the yield of $T_{1}$ is $D$ as required by the context condition of the production $p_{r_{i}-1}$.

The set of context-sensitive syntax trees of $G_{1}$ is defined as

$$
S_{C S}\left(G_{1}\right)=\left\{T \in F_{G_{1}}: S_{1}^{\prime}\left(\rightarrow_{G_{1}[C S]}^{\mathrm{par}}\right)^{*} T\right\},
$$

where $S_{1}^{\prime}$ denotes the tree with one node labeled with the initial nonterminal $S_{1}$. Also define

$$
T S_{C S}\left(G_{1}\right)=S_{C S}\left(G_{1}\right) \cap T S\left(G_{1}\right)
$$

Claim 2. $T S_{C S}\left(G_{1}\right) \neq \emptyset$ if and only if $L\left(G_{P N F}\right) \neq \emptyset$.

Proof of Claim 2. Let $f_{\lambda}:\left(U_{N} \cup U_{T} \cup\{X, Y, \$, @\}\right)^{*} \rightarrow\left(U_{N} \cup U_{T}\right)^{*}$ be the morphism defined by $f_{\lambda}(a)=a$, for $a \in U_{N} \cup U_{T}$, and $f_{\lambda}(X)=f_{\lambda}(Y)=f_{\lambda}(\$)=f_{\lambda}(@)=\lambda$.

First assume that $T S_{C S}\left(G_{1}\right) \neq \emptyset$ and let

$$
T_{0} \rightarrow \underset{G_{1}[C S]}{\mathrm{par}} T_{1} \rightarrow \underset{G_{1}[C S]}{\mathrm{par}} \cdots \rightarrow \rightarrow_{G_{1}[C S]}^{\mathrm{par}} T_{m}
$$

be a parallel context-sensitive derivation of $T_{m} \in T S_{C S}\left(G_{1}\right)$, where $T_{0}$ is the tree with one node labeled by $S_{1}$. From condition (CS) in the definition of the relation $\rightarrow_{G_{1}[C S]}^{\mathrm{par}}$ it follows that

$$
f_{\lambda}\left(\operatorname{yield}\left(T_{i}\right)\right) \Rightarrow_{G_{P N F}}^{*} f_{\lambda}\left(\operatorname{yield}\left(T_{i+1}\right)\right),
$$

$1 \leq i \leq m-1$. Note that the productions of $G_{P N F}$ involve only right context conditions. Hence if we ignore the external nodes labeled by the nonterminal $X$, then a parallel derivation step of $G_{1}$ that satisfies (CS) correctly simulates a sequence of derivation steps of $G_{P N F}$ performed from left-to-right. By (10) it follows that

$$
I=f_{\lambda}\left(\operatorname{yield}\left(T_{1}\right)\right) \Rightarrow_{G_{P N F}}^{*} f_{\lambda}\left(\operatorname{yield}\left(T_{m}\right)\right) \in U_{T}^{+} ;
$$

thus, $L\left(G_{P N F}\right) \neq \emptyset$. 
For the proof in the "if"-direction, assume that

$$
I=w_{0} \Rightarrow_{G_{P N F}} w_{1} \Rightarrow_{G_{P N F}} \cdots \Rightarrow_{G_{P N F}} w_{m} \Rightarrow_{G_{P N F}}^{*} w_{m+1},
$$

where $w_{0}, \ldots, w_{m} \in U_{N}^{+}, w_{m+1} \in U_{T}^{+}$and the derivation $w_{m} \Rightarrow_{G_{P N F}}^{*} w_{m+1}$ uses only the terminating productions of $G_{P N F}$. Since the context conditions of $G_{P N F}$ involve only nonterminals, every string $w_{m+1} \in L\left(G_{P N F}\right)$ has a derivation of this form. We show that, for every $i \in\{0, \ldots, m\}$, there exists $T_{i} \in S_{C S}\left(G_{1}\right)$ such that

$$
f_{\lambda}\left(\operatorname{yield}\left(T_{i}\right)\right)=w_{i} \text { and } \operatorname{yield}\left(T_{i}\right) \in\left(U_{N} \cup\{X, Y\}\right)^{*}
$$

(i) For $i=0$, we choose $T_{0}$ to be the tree obtained from $S_{1}$ with the production $\left(G_{1} \cdot 0\right)$.

(ii) Assume that there is a $T_{i} \in S_{C S}\left(G_{1}\right)$ that satisfies (11), $i<m$. Assume that $w_{i+1}$ is obtained from $w_{i}$ using a production $p_{j}: B D \rightarrow C D,\left(B, C, D \in U_{N}\right), 1 \leq j \leq k_{1}$. The case where the production is context-free is similar and simpler. Let $u$ be the external node of $T_{i}$ that is labeled with the corresponding occurrence of the nonterminal $B$. We construct $T_{i+1}$ by applying, to the external node $u$, the production

$$
B \rightarrow C X^{j}
$$

of $h$ and to all other external nodes appropriate productions $E \rightarrow E, E \in U_{N} \cup\{X, Y\}$. Since $f_{\lambda}\left(\operatorname{yield}\left(T_{i}\right)\right)=w_{i}$, it is clear that this derivation step satisfies the condition (CS).

Since $w_{m}$ can be rewritten to give $w_{m+1}$ using only terminating productions, there exists $T_{m+1} \in$ $T S_{C S}\left(G_{1}\right)$ such that $T_{m} \rightarrow \underset{G_{1}[C S]}{\mathrm{par}} T_{m+1}$ using the productions $\left(G_{1} \cdot 4\right)$ and $X \rightarrow \$, Y \rightarrow @$. This concludes the proof of the claim.

Next, we define the EPT0L grammar $G_{2}$. Intuitively, the grammar $G_{2}$ generates exactly all syntax trees of $G_{1}$ that are not context-sensitive. We augment the nonterminals of $G_{1}$ with additional components that nondeterministically verify that the context condition is violated somewhere in the syntax tree. For this purpose, $G_{2}$ needs more than one table. Let $Z=\left\{z, z_{1}, z_{2}\right\}$ and define the EPT0L grammar $G_{2}=(V, \Sigma, S, H)$, where

- $V=U_{N} \cup\{X, Y\} \cup\left(\left(U_{N} \cup\left\{Y, S_{1}\right\}\right) \times Z\right)$,

- $\Sigma=U_{T} \cup\{\$, @\}$,

- $S=\left(S_{1}, z\right) \in V$, and

- $H=\left\{g, g_{1}, \ldots, g_{k_{1}}\right\}$, where $k_{1}$ is from $(9)$.

The tables $g, g_{1}, \ldots, g_{k_{1}}$ are defined as follows. The table $g$ contains productions $\left(G_{1} .1\right)-\left(G_{1} .5\right)$ of $h$ and additionally the following productions:

$\left(G_{2} .0\right)(\mathbf{i})\left(S_{1}, z\right) \rightarrow(I, z) Y$,

(ii) $\left(S_{1}, z\right) \rightarrow\left(I, z_{1}\right)\left(Y, z_{2}\right)$. 
$\left(G_{2} .1\right)$ If $B \rightarrow C X^{i} \in h, B, C \in U_{N}, 1 \leq i \leq k_{1}$, then

(i) $(B, z) \rightarrow(C, z) X^{i}$,

(ii) $\left(B, z_{j}\right) \rightarrow\left(C, z_{j}\right) X^{i}, j=1,2$.

$\left(G_{2} .2\right)$ If $B \rightarrow C D X^{i} \in h, B, C, D \in U_{N}, k_{1}<i \leq k$, then

(i) $(B, z) \rightarrow(C, z) D X^{i},(B, z) \rightarrow C(D, z) X^{i}$,

(ii) $(B, z) \rightarrow\left(C, z_{1}\right)\left(D, z_{2}\right) X^{i}$,

(iii) $\left(B, z_{1}\right) \rightarrow C\left(D, z_{1}\right) X^{i}$,

(iv) $\left(B, z_{2}\right) \rightarrow\left(C, z_{2}\right) D X^{i}$.

$\left(G_{2} . \mathbf{3}\right)$ For every $B \in U_{N}$, the productions $(B, z) \rightarrow(B, z),\left(B, z_{j}\right) \rightarrow\left(B, z_{j}\right), j=1,2$, and, $\left(Y, z_{2}\right) \rightarrow\left(Y, z_{2}\right)$.

Let $r \in\left\{1, \ldots, k_{1}\right\}$ and assume that the right-context production $p_{r}$ of $G_{P N F}$ is of the form

$$
p_{r}: B D \rightarrow C D, \quad\left(B, C, D \in U_{N}\right) .
$$

The table $g_{r}$ contains the productions $\left(G_{1} .1\right)-\left(G_{1} .5\right)$ of $h$ and the productions

$\left(M_{1}^{r}\right)\left(B, z_{1}\right) \rightarrow C X^{r}$.

$\left(M_{2}^{r}\right)\left(E, z_{2}\right) \rightarrow w$, where $E \in\left(U_{N}-\{D\}\right) \cup\{Y\}, w \in\left(U_{N} \cup\{X, Y\}\right)^{+}$and $E \rightarrow w \in h$.

Let $f_{Z}:(V \cup \Sigma)^{*} \rightarrow\left(U_{N} \cup U_{T} \cup\left\{X, Y, S_{1}, \$, @\right\}\right)^{*}$ be the morphism determined by the conditions: $f_{Z}((x, y))=x, x \in U_{N} \cup\left\{Y, S_{1}\right\}, y \in Z$, and $f_{Z}(x)=x$ when $x \in U_{N} \cup U_{T} \cup\{X, Y, \$, @\}$. The function $f_{Z}$ simply erases the second components belonging to $Z$ from the nonterminals. Then every production $L \rightarrow R$ belonging to the tables $g, g_{1}, \ldots, g_{k_{1}}$ has the property that

$$
f_{Z}(L) \rightarrow f_{Z}(R) \in h
$$

If $T \in S\left(G_{2}\right)$, we denote by $f_{Z}(T)$ the tree defined by the conditions $\operatorname{dom}\left(f_{Z}(T)\right)=\operatorname{dom}(T)$, and $\operatorname{lab}_{f_{Z}(T)}(u)=f_{Z}\left(\operatorname{lab}_{T}(u)\right), u \in \operatorname{dom}(T)$. It follows by $(13)$ that

$$
\left(\forall T \in S\left(G_{2}\right)\right) f_{Z}(T) \in S\left(G_{1}\right) .
$$

Hence it follows also that

$$
\operatorname{STS}\left(G_{2}\right) \subseteq S T S\left(G_{1}\right)
$$

Intuitively, the symbols $z, z_{1}, z_{2}$ can be seen as messengers that travel nondeterministically down in a syntax tree of $G_{1}$ and find a position where the syntax tree violates the context condition (CS). In a sentential form of $G_{2}$ the messengers $z_{1}$ and $z_{2}$ will always be forced to be located in nonterminals $N_{1}, N_{2} \in U_{N} \cup\{Y\}$ that are separated only by a sequence of nonterminals $X$. Thus $N_{1}$ and $N_{2}$ represent consecutive nonterminals in the derivation of $G_{P N F}$ that is simulated or $N_{1}$ is the rightmost nonterminal in the derivation of $G_{P N F}$ and $N_{2}=Y$ is the "right endmarker." The 
tables of $G_{2}$ are defined so that the only possibility to delete the symbols $z_{1}$ and $z_{2}$ is to apply productions of a table $g_{r}, 1 \leq r \leq k_{1}$, that force the context condition to be violated.

In the following we show that $G_{2}$ generates exactly the structures of syntax trees of $G_{1}$ that do not correspond to a context-sensitive syntax tree.

Claim 3. $S T S\left(G_{2}\right)=\operatorname{STS}\left(G_{1}\right)-\operatorname{str}_{G_{1}}\left(T S_{C S}\left(G_{1}\right)\right)$.

Proof of Claim 3. Let $t \in S T S\left(G_{1}\right)-\operatorname{str}_{G_{1}}\left(T S_{C S}\left(G_{1}\right)\right)$. By Claim 1, there exists a unique $T \in T S\left(G_{1}\right)-T S_{C S}\left(G_{1}\right)$ such that $\operatorname{str}_{G_{1}}(T)=t$. Denote the parallel derivation sequence of $T$ by

$$
T_{0} \rightarrow{ }_{G_{1}}^{\mathrm{par}} T_{1} \rightarrow{ }_{G_{1}}^{\mathrm{par}} \cdots \rightarrow \rightarrow_{G_{1}}^{\mathrm{par}} T_{m}=T,
$$

where $T_{0}$ is the tree with one node labeled by $S_{1}$. (Note that given $T$ the derivation sequence (15) is uniquely determined.) Since $T \notin T S_{C S}\left(G_{1}\right)$, there exists $i \in\{1, \ldots, m-1\}$ such that the derivation step

$$
T_{i} \rightarrow{ }_{G_{1}}^{\mathrm{par}} T_{i+1}
$$

does not satisfy the condition (CS). Thus in $T_{i}$ there exists an external node $u$ labeled with $A \in U_{N}$ such that in the derivation step (16) at $u$ we apply a production

$$
A \rightarrow C X^{j} \in h,
$$

$1 \leq j \leq k_{1}$, the next external node $u^{\prime}$ of $T_{i}$ to the right from $u$ that is labeled by an element different from $X$ is labeled with $E \in U_{N} \cup\{Y\}$, and the production $p_{j}$ of $G_{P N F}$ has the form $A D \rightarrow C D$, where $D \neq E$. Denote by $u_{0}$ the least common predecessor of $u$ and $u^{\prime}$; that is, $u_{0}$ is the common predecessor of $u$ and $u^{\prime}$ furthest from the root of $T_{i}$. Let the distance of $u_{0}$ from the root be $e$, that is, $u_{0}$ is an external node of $T_{e}, 0 \leq e<i$. We construct a derivation sequence of $G_{2}$

$$
\left(S_{1}, z\right)=T_{0}^{\prime} \rightarrow_{G_{2}}^{\mathrm{par}} T_{1}^{\prime} \rightarrow_{G_{2}}^{\mathrm{par}} \cdots \rightarrow_{G_{2}}^{\mathrm{par}} T_{m}^{\prime}
$$

as follows. The first components of the nonterminals in the derivation (18) simulate directly the derivation (15), that is, $f_{Z}\left(T_{c}^{\prime}\right)=T_{c}, c=0, \ldots, m$. The first $i$ steps of (18) use only the table $g$. In the first $e$ derivation steps the messenger symbol $z$ travels nondeterministically to the external node $u_{0}$ of $T_{e}^{\prime}$ using productions $\left(G_{2} \cdot 0\right)(\mathrm{i}),\left(G_{2} \cdot 1\right)(\mathrm{i}),\left(G_{2} \cdot 2\right)(\mathrm{i})$ and $\left(G_{2} \cdot 3\right)$. The external node $u_{0}$ is in the natural way viewed also as a node of $T_{e}^{\prime}$. In the following we always identify the corresponding nodes of $T_{c}$ and $T_{c}^{\prime}, c \in\{0, \ldots, m\}$. Since $u_{0}$ is the least common predecessor of $u$ and $u^{\prime}$, it follows that necessarily the production applied at $u_{0}$ in $(15)$ is either $\left(G_{1} .0\right)$ or of the type $\left(G_{1} .2\right)$. (The productions $\left(G_{1} .1\right)$ and $\left(G_{1} .3\right)$ have only one successor labeled by an element of $U_{N} \cup\{Y\}$.) In the derivation step $T_{e}^{\prime} \rightarrow_{G_{2}}^{\mathrm{par}} T_{e+1}^{\prime}$ at the node $u_{0}$ we use the corresponding production $\left(G_{2} .0\right)$ (ii) or $\left(G_{2} .2\right)$ (ii) that branches the $z$-messenger into the messengers $z_{1}$ and $z_{2}$. By the definition of the productions $\left(G_{2} .1\right)(\mathrm{ii}),\left(G_{2} \cdot 2\right)$ (iii),(iv) and $\left(G_{2} .3\right)$ it is clear that in the tree $T_{i}^{\prime}$ the $z_{1}$-messenger has reached the node $u$ and the $z_{2}$-messenger is in the node $u^{\prime}$. Note that in productions $\left(G_{2} .2\right)(\mathrm{iii})$ and (iv) the $z_{1}$-messenger always follows the rightmost branch not consisting of $X$-nonterminals and the symbol $z_{2}$ always follows the leftmost branch. These paths are just the paths from $u_{0}$ to the nodes $u$ and $u^{\prime}$, since $u$ and $u^{\prime}$ are consecutive external nodes of $T_{i}$ when we disregard nodes labeled by the $X$-nonterminals. 
Thus, the node $u$ in $T_{i}^{\prime}$ is labeled by $\left(A, z_{1}\right)$ and the node $u^{\prime}$ is labeled by $\left(E, z_{2}\right)$; see $(17)$. The derivation step $T_{i}^{\prime} \rightarrow{ }_{G_{2}}^{\mathrm{par}} T_{i+1}^{\prime}$ uses the table $g_{j}$ to eliminate the messengers $z_{1}$ and $z_{2}$ by productions $\left(M_{1}^{j}\right)$ and $\left(M_{2}^{j}\right)$. (Here $j$ is from (17).) At external nodes of $T_{i}^{\prime}$ other than $u$ and $u^{\prime}$, we apply the same productions as in (16), which is possible, since the table $g_{j}$ contains the productions $\left(G_{1} .1\right)-\left(G_{1} .5\right)$.

Now, $\operatorname{yield}\left(T_{i+1}^{\prime}\right)=\operatorname{yield}\left(T_{i+1}\right)$ and the derivation (18) can be completed as in (15); hence, $t=\operatorname{str}_{G_{2}}\left(T_{m}^{\prime}\right), T_{m}^{\prime} \in T S\left(G_{2}\right)$ implies that $t \in S T S\left(G_{2}\right)$.

For the converse, let $t \in S T S\left(G_{2}\right)$ and assume that $t=\operatorname{str}_{G_{2}}(T), T \in T S\left(G_{2}\right)$. By (14), $f_{Z}(T) \in T S\left(G_{1}\right)$; thus, $t=\operatorname{str}_{G_{1}}\left(f_{Z}(T)\right) \in S T S\left(G_{1}\right)$. Let

$$
\left(S_{1}, z\right)=T_{0} \rightarrow G_{G_{2}}^{\mathrm{par}} T_{1} \rightarrow{ }_{G_{2}}^{\mathrm{par}} \cdots \rightarrow \rightarrow_{G_{2}}^{\mathrm{par}} T_{m}=T
$$

be a derivation of $T$. Since $T$ is a terminal syntax tree, in some step $T_{i} \rightarrow{ }_{G_{2}}^{\mathrm{par}} T_{i+1}$ of (19), $0 \leq i<m-1$, we have to divide the messenger $z$ to the pair of messengers $z_{1}$ and $z_{2}$ that are then finally destroyed by productions $\left(M_{1}^{r}\right)$ and $\left(M_{2}^{r}\right)$ of a suitable table $g_{r}, 1 \leq r \leq k_{1}$, in a derivation step $T_{j} \rightarrow{ }_{G_{2}}^{\mathrm{par}} T_{j+1}, i<j \leq m-1$. (There is no other way to delete the messenger symbols.) It is easy to see inductively that, for all $n \in\{i+1, \ldots, j\}$, we can write

$$
\operatorname{yield}\left(T_{n}\right)=w_{1}\left(A_{1}, z_{1}\right) X^{s}\left(A_{2}, z_{2}\right) w_{2},
$$

where $A_{1} \in U_{N}, A_{2} \in U_{N} \cup\{Y\}, w_{1} \in\left(U_{N} \cup\{X\}\right)^{*}, w_{2} \in\left(U_{N} \cup\{X, Y\}\right)^{*}$, and $s \geq 0$; that is, the messenger symbols $z_{1}$ and $z_{2}$ label consecutive nonterminals in the yield when we disregard nodes labeled by $X$. From the form of the productions $\left(M_{1}^{r}\right)$ and $\left(M_{2}^{r}\right)$, the derivation step $f_{Z}\left(T_{j}\right) \rightarrow_{G_{1}}^{\text {par }}$ $f_{Z}\left(T_{j+1}\right)$ does not satisfy the condition (CS); thus, $f_{Z}(T) \notin T S_{C S}\left(G_{1}\right)$. Since $\operatorname{str}_{G_{1}}$ is injective, we deduce that $t \notin \operatorname{str}_{G_{1}}\left(T S_{C S}\left(G_{1}\right)\right)$, which completes the proof of Claim 3.

Combining Claims 2 and 3, we obtain $S T S\left(G_{1}\right)=S T S\left(G_{2}\right)$ if and only if $L\left(G_{P N F}\right)=\emptyset$. By Theorem 2.1, this implies that (8) is undecidable in general.

The following simple example illustrates the construction of the proof of Theorem 4.1.

Example 4.1 Consider the PNF grammar $G_{P N F}=\left(U_{N}, U_{T}, I, P\right)$ where $U_{N}=\{I, A, B, C\}$, $U_{T}=\{a, b\}$ and $P$ consists of the right-context production $p_{1}: C B \rightarrow A B$, the context-free productions $p_{2}: I \rightarrow C A, p_{3}: I \rightarrow C B$ and the terminating productions $A \rightarrow a, B \rightarrow b .\left(p_{1}, p_{2}\right.$, $p_{3}$ are the names for the nonterminating productions as used in the proof of Theorem 4.1.)

Let $G_{1}$ and $G_{2}$ be the EP(T)0L grammars constructed from $G_{P N F}$ as in the proof of Theorem 4.1. The grammar $G_{1}$ has for instance the following parallel derivation of a sentence:

$$
S_{1} \Rightarrow_{G}^{\mathrm{par}} I Y \Rightarrow_{G}^{\mathrm{par}} C A X X Y \Rightarrow_{G}^{\mathrm{par}} A X A X X Y \Rightarrow_{G}^{\mathrm{par}} a \$ a \$ \$ \text {. }
$$

(In the nonterminating parallel steps we always apply to the nonterminals $X$ and $Y$ the productions $X \rightarrow X, Y \rightarrow Y$.) In the third parallel derivation step the grammar $G_{1}$ rewrites the leftmost nonterminal $C$ by a production simulating $p_{1}$ but ignoring the right-context condition. Thus $G_{2}$ can simulate the derivation $(20)$ as follows:

$$
\left(S_{1}, z\right) \Rightarrow_{G}^{\mathrm{par}}(I, z) Y \Rightarrow_{G}^{\mathrm{par}}\left(C, z_{1}\right)\left(A, z_{2}\right) X X Y \Rightarrow_{G}^{\mathrm{par}} A X A X X Y \Rightarrow_{G}^{\mathrm{par}} a \$ a \$ \$ @ \text {. }
$$


In the third parallel step of (21) $G_{2}$ uses the table $g_{1}$ that verifies that the context condition is violated in the consecutive nonterminals $\left(C, z_{1}\right)$ and $\left(A, z_{2}\right)$. It is clear that the structures of the syntax trees corresponding to the derivations (20) and (21) are identical.

However, $L\left(G_{P N F}\right)$ is nonempty and the EP0L grammar $G_{1}$ has the following parallel derivation simulating a correct context-sensitive derivation of $G_{P N F}$ :

$$
S_{1} \Rightarrow \underset{G}{\mathrm{par}} I Y \Rightarrow_{G}^{\mathrm{par}} C B X X X Y \Rightarrow_{G}^{\mathrm{par}} A X B X X X Y \Rightarrow_{G}^{\mathrm{par}} a \$ b \$ \$ \$ @
$$

The EPT0L grammar $G_{2}$ does not have any derivation with the same structure as the preceding derivation. (If $G_{2}$ attempts to "simulate" (22) it cannot get rid of the $z$-symbols.) Thus $G_{1}$ and $G_{2}$ are not structurally equivalent as required since $L\left(G_{P N F}\right) \neq \emptyset$.

The contrasting results of Theorems 3.1 and 4.1 can be interpreted by saying that, at least in the ET0L case, one loses essential information about a derivation when going from syntax trees to the corresponding structure trees.

In the proof of Theorem 4.1, the number of tables of the EPT0L grammar $G_{2}$ depends on the PNF context-sensitive grammar $G_{P N F}$. Every ET0L grammar is language equivalent to an ET0L grammar that has only two tables [11], but the corresponding transformation clearly does not preserve structural equivalence of the grammars. We can, however, strengthen Theorem 4.1 somewhat.

Theorem 4.2 Given an EPOL grammar $G_{1}$ and an EPTOL grammar $G_{2}$ that has two tables it is undecidable whether

$$
\operatorname{STS}\left(G_{1}\right)=\operatorname{STS}\left(G_{2}\right)
$$

Proof. Given a PNF context-sensitive grammar $G_{P N F}$ we construct the grammar $G_{1}$ exactly as in the proof of Theorem 4.1 and transform the grammar $G_{2}$ into a grammar $G_{2}^{\prime}$ that has two tables as follows. In $G_{2}^{\prime}$, we merge the tables $g_{1}, \ldots, g_{k_{1}}$ into one table by coding, in the messenger symbols, the information about the production of $G_{P N F}$ whose context condition the derivation is going to violate. When the messenger $z$ branches into two messengers using the production $\left(G_{2} \cdot 2\right)$ (ii) or $\left(G_{2} .0\right)$ (ii), the grammar chooses, nondeterministically, a pair of messengers $z_{1}^{r}, z_{2}^{r}, 1 \leq r \leq k_{1}$. The first table of $G_{2}^{\prime}$ is essentially the table $g$ augmented with the preceding nondeterministic choice. The second table $g^{\prime}$ contains the productions $\left(G_{1} .1\right)-\left(G_{1} .5\right)$ of $h$ and, for every $r \in\left\{1, \ldots, k_{1}\right\}$ and $p_{r}$ of $G_{P N F}$ of the form given in (12), $g^{\prime}$ contains the productions

- $\left(B, z_{1}^{r}\right) \rightarrow C X^{r}$.

- $\left(E, z_{2}^{r}\right) \rightarrow w$, where $E \in\left(U_{N}-\{D\}\right) \cup\{Y\}, w \in\left(U_{N} \cup\{X, Y\}\right)^{+}$and $(E \rightarrow w) \in h$.

Intuitively, in the syntax trees of $G_{2}^{\prime}$, we determine which of the tables $g_{1}, \ldots, g_{k_{1}}$ will be used to delete the messengers when we choose the symbols $z_{1}^{r}$ and $z_{2}^{r}, 1 \leq r \leq k_{1}$. It is clear that $S T S\left(G_{2}^{\prime}\right)=S T S\left(G_{2}\right)$; therefore, we cannot decide whether $G_{1}$ and $G_{2}^{\prime}$ are structurally equivalent. 
Theorem 4.2 is optimal with respect to the number of tables, since structural equivalence is decidable for E0L grammars. On the other hand, it is clear that the proof method of Theorem 4.1 does not work if the tables of a ET0L grammar are homomorphisms; that is, we have EDT0L grammars [11]. It is an open question whether structural equivalence is decidable for EDT0L grammars.

\section{References}

[1] H. A. Cameron and D. Wood, Structural equivalence of extended context-free and E0L grammars, unpublished manuscript, 1995.

[2] S. Ginsburg and M. Harrison, Bracketed context-free languages, Journal of Computer and System Sciences 1 (1967), 1-23.

[3] G. Istrate, The strong structural equivalence of ET0L grammars. In: "Developments in Language Theory," G. Rozenberg and A. Salomaa (eds.), World Scientific Publishing, Singapore, 1994, pp. 81-89.

[4] N. D. Jones and S. Skyum, Complexity of some problems concerning L systems, Mathematical Systems Theory 13 (1979), 29-43.

[5] R. McNaughton, Parenthesis grammars, Journal of the Association of Computing Machinery 14 (1967), 490-500.

[6] V. Niemi, A normal form for structurally equivalent E0L grammars. In: "Lindenmayer Systems: Impacts on Theoretical Computer Science, Computer Graphics, and Developmental Biology", G. Rozenberg and A. Salomaa (eds.), Springer-Verlag, 1992, pp. 133-148.

[7] Th. Ottmann and D. Wood, Defining families of trees with E0L grammars, Discrete Applied Mathemtaics 32 (1991), 195-209.

[8] Th. Ottmann and D. Wood, Simplifications of E0L grammars. In: "Lindenmayer Systems: Impacts on Theoretical Computer Science, Computer Graphics, and Developmental Biology", G. Rozenberg and A. Salomaa (eds.), Springer-Verlag, 1992, pp. 149-166.

[9] M. Paull and S. Unger, Structural equivalence of context-free grammars, Journal of Computer and System Sciences 2 (1968), 427-463.

[10] M. Penttonen, One-sided and two-sided context in formal grammars, Information and Control 25 (1974), 371-392.

[11] G. Rozenberg and A. Salomaa, The Mathematical Theory of L Systems. Academic Press, New York, 1980.

[12] A. Salomaa, Formal Languages. Academic Press, New York, 1973. 
[13] K. Salomaa, D. Wood, and S. Yu, Complexity of E0L structural equivalence, RAIRO Informatique théorique et Applications (1995), to appear.

[14] K. Salomaa and S. Yu, Decidability of structural equivalence of E0L grammars, Theoretical Computer Science 82 (1991), 131-139.

[15] J. W. Thatcher, Characterizing derivation trees of a context-free grammar through a generalization of finite-automata theory, Journal of Computer and System Sciences 1 (1967), $317-322$.

[16] J. W. Thatcher, Tree automata: an informal survey. In: "Currents in the Theory of Computing", A. V. Aho (ed.), Prentice Hall, Englewood Cliffs, NJ, 1973, pp. 143-172.

[17] D. Wood, Theory of Computation. John Wiley \& Sons, New York, 1987.

[18] D. Wood, Standard Generalized Markup Language: Mathematical and philosophical issues. In: "Computer Science Today," J. van Leeuwen (ed.), Springer-Verlag Lecture Notes in Computer Science 1000 (1995), to appear. 